\title{
Autonomous Formation Flight
}

\section{A primary goal is to reduce fuel consumption during cruise by 10 percent.}

\section{Dryden Flight Research Center, Edwards, California}

NASA's Strategic Plan for the Aerospace Technology Enterprise includes ambitious objectives focused on affordable air travel, reduced emissions, and expanded aviation-system capacity. NASA Dryden Flight Research Center, in cooperation with NASA Ames Research

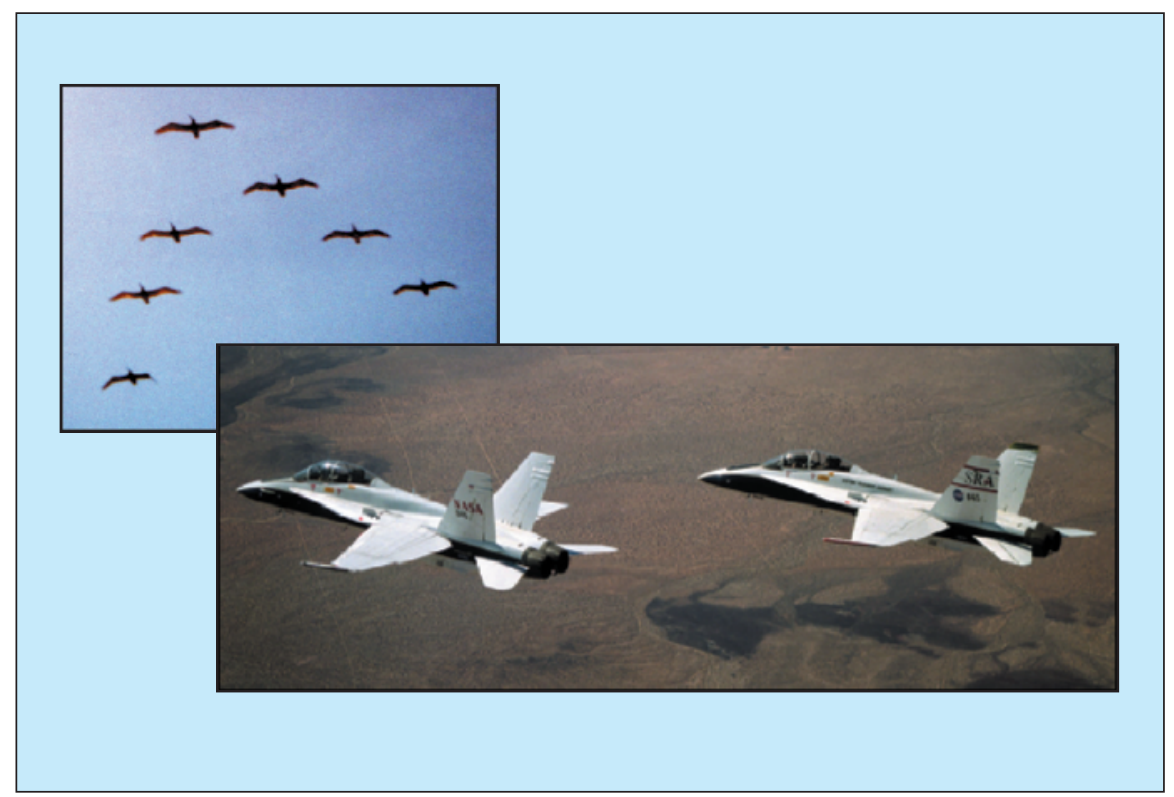

Figure 1. Formation Flight offers the advantage of reduced overall drag for both birds and airplanes.

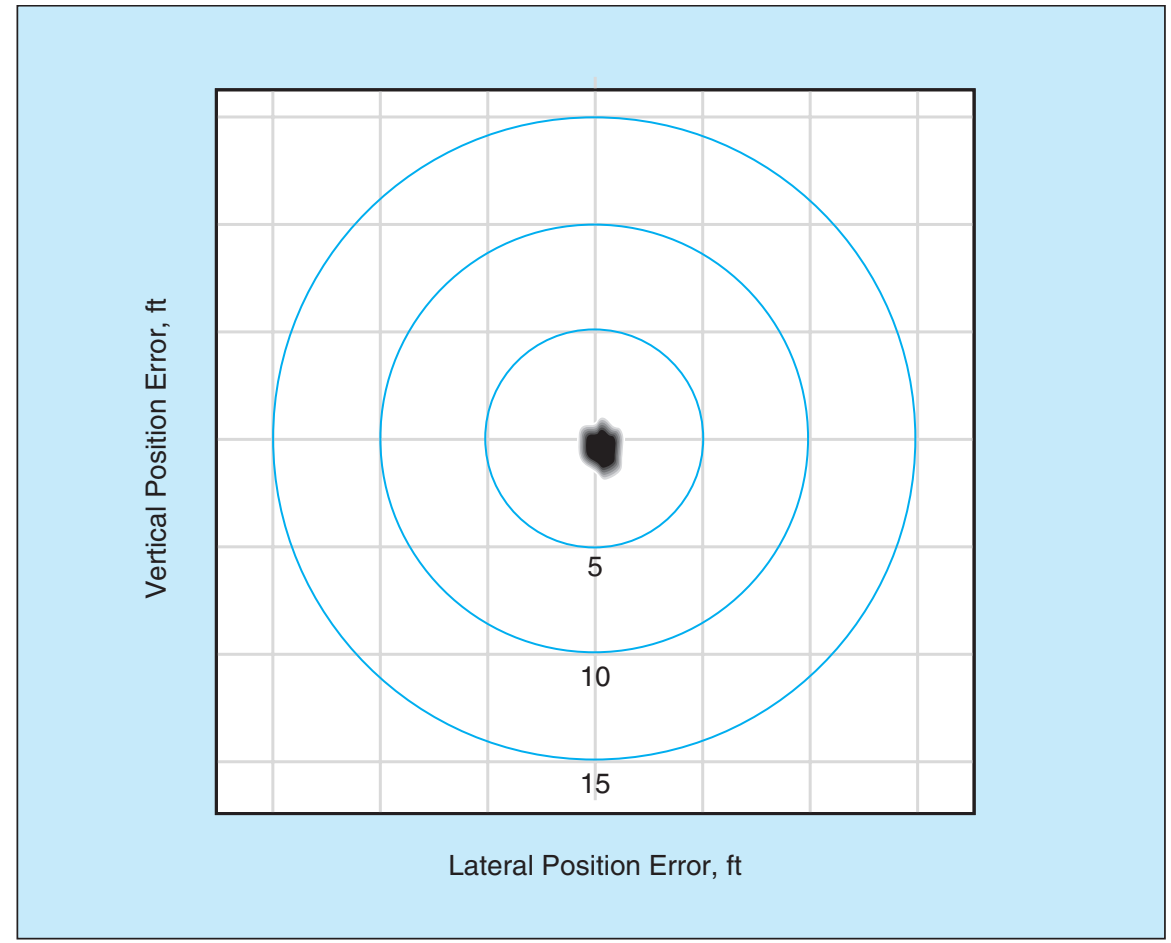

Figure 2. The Narrowness of the Scatter of relative-position measurements taken in flight tests is proof of the viability of a formation autopilot system. flying in formation. The traditional "V" formation flown by many species of birds (including gulls, pelicans, and geese) enables each of the trailing birds to fly in the upwash flow field that exists just outboard of the bird immediately ahead in the formation. The result for each trailing bird is a decrease in induced drag and thus a reduction in the energy needed to maintain a given speed. Hence, for migratory birds, formation flight extends the range of the system of birds over the range of birds flying solo. The Autonomous Formation Flight (AFF) Project is seeking to extend this symbiotic relationship to aircraft (see Figure 1).

Predicted benefits of AFF as applied to commercial transport airplanes for typical transcontinental routes include annual per-trailing-airplane reductions of $\$ 0.5 \times 10^{6}$ (year 2000 average prices) in the cost of fuel, $10^{7} \mathrm{lb}\left(\approx 4.5 \times 10^{6} \mathrm{~kg}\right)$ in emitted carbon dioxide, and $10^{5} \mathrm{lb}$ $\left(\approx 4.5 \times 10^{4} \mathrm{~kg}\right)$ in emitted nitrous oxide. In addition, improvements in cooperative guidance and control could one day enable air-traffic-control systems to manage formations of aircraft as though they were single aircraft, thereby increasing overall throughput.

AFF was competitively selected in May 2000 by the Revolutionary Concepts in Aeronautics (RevCon) project, funded under Dryden Flight Research Centers's R\&T Base Program. RevCon was designed to accelerate, through flight research, the dissemination of new aircraft and system concepts.

The primary goal of the AFF project is to demonstrate a sustained 10-percent reduction in the consumption of fuel by a trailing airplane during cruise. The project intends to advance the concept of autonomous-formation-flight drag reduction from the experimental proof-of-concept stage to a prototype demonstration within three years. The prototype demonstration will be accomplished by use of two highly instrumented NASA F/A-18 aircraft equipped with the necessary research systems.

The AFF project will involve three phases, with flight tests beginning in the first quarter of fiscal year 2001 and completion by the end of fiscal year 2003. The first phase, which has taken place, was devoted to the demonstra- 
tion of precise autonomous station keeping. The second phase, also completed, involved mapping of the effects of leading-airplane-generated vortices on a evaluation of the performances of a highly accurate relative-position-sensing system and of a data-link system. The primary goal of the project demonstration of integrated system performance, including a sustained 10percent reduction in fuel consumption in close, autonomous formation flight - will be reached in the third phase. The results of this phase will be applicable to commercial and military cargo and passenger transport aircraft, unpiloted aircraft, increasing the ranges of aircraft in general, aerial refueling and resupply, and formation flying of satellites.

The AFF project is developing the first system for highly accurate in-flight relative positioning of two aircraft to incorporate differential-carrier-phase Global Positioning System (GPS) and inertial measurement information with an extended Kalman filter in a movingbase-station scenario. This system is expected to yield relative-position measurements accurate to within $0.5 \mathrm{ft}$ $(0.15 \mathrm{~m})$. Flight tests will enable evaluation of control-system approaches and performance, validation of mathematical models for predicting vortex effects, quantification of reductions of drag, and evaluation of operational effectiveness. The flight tests will provide insight into such phenomena as effects of multipath propagation on GPS measurements and data communications and the dynamics of (including interactions between) vortices that cannot be adequately identified through simulation or ground test.

During the first phase of the program, two F/A-18 airplanes were outfitted with identical GPS receivers and an air-to-air telemetry system as a data link between the airplanes. In addition, the trailing airplane was equipped with an airborne research test system (ARTS) and a research flight-control computer. The ARTS hosted a precise autopilot control system, which received GPS and inertial measurement data from the leading airplane and computed stick commands to place the trailing airplane at the desired relative position. The research flight-control computer received and used the stick commands from the ARTS while engaged, but reverted to the production F-18 flight control system for the remainder of the mission. An interactive display was installed in the back seat of the airplane to enable the flight crew to control the flight experiment. Lateral and vertical position errors were displayed to the pilot by use of instrument-landing-system needles.
A standard test block of six maneuvers was repeated for each of four different autopilot gain sets. These maneuvers included five-minute steady-state tracking tests and $30-\mathrm{ft}(\approx 9-\mathrm{m})$ commanded step inputs in each axis. The dynamic response of the system was observed in maneuvers in which the leading airplane performed heading sweeps of a few degrees and altitude sweeps of several hundred feet $(\approx 100 \mathrm{~m})$.

A total of 167 tests points was reached in 11 research flights. The experiment met all project objectives. The formation autopilot maintained relative position to within $2 \mathrm{ft}(0.61 \mathrm{~m})$ [see Figure 2] for all four gain sets during straight and level flight with turbulence levels ranging from nonexistent to light chop. An additional position error of up to $3 \mathrm{ft}(0.91$ $\mathrm{m})$, due to GPS navigation errors, brought the total formation position error to less than $5 \mathrm{ft}(1.52 \mathrm{~m})$. Accurate, predictable tracking was observed during the step and dynamic maneuvers.

This work was done by Gerard S. Schkolnik and Brent Cobleigh of Dryden Flight Research Center. For more information on the AFF project contact Gerard S. Schkolnik, AFF Project Manager, gerard.schkolnik@ dfrc.nasa.gov, (661) 276-3055 or Brent Cobleigh, AFF Chief Engineer, brent.cobleigh@ dfrc.nasa.gov, (661) 276-2249.

DRC-01-46

\section{Expandable Purge Chambers Would Protect Cryogenic Fittings Flowing dry nitrogen would prevent accumulation of ice or airborne particles.}

\section{John F. Kennedy Space Center, Florida}

Expandable ice-prevention and cleanliness-preservation (EIP-CP) chambers have been proposed to prevent the accumulation of ice or airborne particles on quick-disconnect (QD) fittings, or on ducts or tubes that contain cryogenic fluids. In the original application for which the EIP-CP chambers were conceived, there is a requirement to be able to disconnect and reconnect the QD fittings in rapid succession. If ice were to form on the fittings by condensation and freezing of airborne water vapor on the cold fitting surfaces, the ice could interfere with proper mating of the fittings, making it necessary to wait an unacceptably long time for the ice to thaw before attempting reconnection. By keeping water vapor away from the cold fitting surfaces, the EIP-CP chambers would prevent accumulation of ice, preserving the ability to reconnect as soon as required.

Basically, the role of an EIP-CP chamber would be to serve as an enclosure for a flow of dry nitrogen gas that would keep ambient air away from QD cryogenic fittings. An EIP-CP chamber would be an inflatable device made of a fabriclike material. The chamber would be attached to an umbilical plate holding a cryogenic QD fitting. The chamber would include inner and outer subchambers that would be inflated with gaseous nitrogen through separate supply tubes. The outer subchamber would resemble a small tire tube. The inner chamber would be perforated on its innermost circle to allow nitrogen to flow onto and around the QD surfaces. When deflated, the EIP-CP would be about 1 in. $(\approx 2.5 \mathrm{~cm})$ thick.

When not in use, the EIP-CP would be kept deflated, flat against the umbilical plate. Before disconnecting the QD fitting, the two subchambers of the EIPCP would be pressurized with nitrogen. As disconnection proceeded, the pressurized outer tube would expand to follow the moving umbilical plate of the mating fitting, up to a maximum axial thickness (corresponding to a tire width) of about 6 in. $(\approx 15 \mathrm{~cm})$. The subchambers would be shaped so that once maximum expansion was reached and the chamber could no longer seal against the receding umbilical plate of the mating fitting, the opening on the exposed end of the chamber would narrow to a small hole. The purge flow of 